\title{
Proton auroral intensifications and injections at synchronous altitude
}

\author{
Eric C. Chi, ${ }^{1}$ Stephen B. Mende, ${ }^{1}$ M.-C. Fok, ${ }^{2}$ and G. D. Reeves ${ }^{3}$ \\ Received 14 September 2005; revised 27 January 2006; accepted 6 February 2006; published 18 March 2006.
}

[1] In sudden flux increases at synchronous altitude the lower energy channels often show progressively more delay or dispersion. It is usually assumed that the dispersion is caused by a simultaneous injection of particles of all energies at some location, and by the subsequent drift of these particles to the synchronous altitude measurement site "downstream" of the injection event. In this paper we present a method for timing and locating the injections from proton auroral precipitation inferred by the Lyman $\alpha$ emission data from the IMAGE FUV instrument. We compare the timing of the proton flux increases observed by the Los Alamos National Laboratory synchronous altitude satellites to the time delay predicted by a model describing the longitudinal drift of particles in the magnetosphere. We present comparisons for eleven substorm particle injections and find that the observed azimuthal drift times are reasonably consistent with those calculated by a simple model using the Tsyganenko 89 magnetic and Volland electric field models as input. This consistency supports the concept that the proton auroral intensification at substorm onset and the proton injection in the magnetosphere occur at the same magnetic local time (longitude). Citation: Chi, E. C., S. B. Mende, M.-C. Fok, and G. D. Reeves (2006), Proton auroral intensifications and injections at synchronous altitude, Geophys. Res. Lett., 33, L06104, doi:10.1029/2005GL024656.

\section{Introduction}

[2] It has been known for some time that sudden increases of particle fluxes observed by satellites in the nightside magnetosphere are associated with the substorm expansion phase. Satellite particle flux intensifications characteristics of such onsets normally exhibit energy dispersion i.e. the intensification of higher energy particle fluxes precedes the lower energy particles. This can be explained in terms of the longer drift times for low energy (slower) particles from injection source to the satellite. Tracing back the particles from their dispersion properties to the injection point was used by several authors [McIlwain, 1974; Reeves et al., 1991, 1992] by assuming that the magnetic and electric fields are time stationary in the period following the injection events. The model of McIlwain [1974] and Lopez et al. [1990] interpreted the injection boundary as being a demarcation between the stable inner and the tailward unstable regions. It is assumed that tailward of this boundary all electrons and protons are energized at the same time at substorm onset, and the particle signatures subse-

\footnotetext{
${ }^{1}$ Space Sciences Laboratory, University of California, Berkeley, California, USA.

${ }^{2}$ NASA Goddard Space Flight Center, Greenbelt, Maryland, USA.

${ }^{3}$ Los Alamos National Laboratory, Los Alamos, New Mexico, USA.
}

Copyright 2006 by the American Geophysical Union. 0094-8276/06/2005GL024656\$05.00 quently observed by satellites show the dispersion due to their energy dependent drift speeds. The location or shape of the injection boundary was assumed to be an extensive region in local time [Mauk and Meng, 1983]. The extent of the injection region near onset is an important parameter because longitudinally localized injection regions support substorm models with a localized onset for example the Near Earth Current Disruption model [Lui, 1988] or flow burst related auroral intensifications [Nakamura et al., 2001] whereas the wide extensive injection front would support models in which large regions of the tail are suddenly populated with energetic particles such as the classical Near Earth Neutral Line model of Hones [1979].

[3] Reeves et al. [1991] noted that dispersionless injections of ions may occur without accompanying electron injection and vice versa. Their results were consistent with test particle simulations of Birn et al. [1998] showing two separate azimuthally separated injection fronts for ions and electrons propagating earthward. If the inward propagation of the injection front takes some time then the satellite would not see them simultaneously due to their azimuthal separation related to the differential drift between electrons and ions [Reeves, 1998]. The simulations of Li et al. [1998] show that at the time of substorm onset an inward propagating compressional wave from the tail would locally reverse the B gradient and inhibit the normal static field induced drifts. Understanding the relationship between the injected ions and the substorm related ion precipitation would provide a significant tool for tracking the spatial dynamics of the injection regions from optical signatures.

[4] In this paper we combine the optically measured proton aurora by the FUV instrument on the NASA IMAGE satellite with synchronous altitude particle measurements. At the time of substorm onsets ( $\mathrm{T}_{\text {aurora }}$ ) the precipitating proton fluxes increase by about a factor of 2 at a spatially localized region [Mende et al., 2003]. We assume that this is the region where the energetic protons are first injected. Assuming that after injection the particles drift under the influence of the magnetospheric fields we calculate the time $\left(\Delta \mathrm{T}_{\text {calc }}\right)$ the particles of different energy would reach regions where the synchronous altitude satellites are located. The particle intensifications are actually seen in the satellite data at $\mathrm{T}_{\text {sat }}$ and we compare $\mathrm{T}_{\text {sat }}-\mathrm{T}_{\text {aurora }}$ to $\Delta \mathrm{T}_{\text {calc }}$.

\section{Observations}

[5] Since 1989, Los Alamos National Laboratory (LANL), has measured energetic particle fluxes in situ in the near-Earth equatorial region in geosynchronous orbit at a nominal radial distance of $6.6 \mathrm{R}_{\mathrm{E}}$, geographic latitude of approximately 0 degrees, and a fixed longitude. The SOPA detector measures electrons from $50 \mathrm{keV}$ to approximately $26 \mathrm{MeV}$, ions from $50 \mathrm{keV}$ to $50 \mathrm{MeV}$, and heavier ions in various channels with energies in the $\mathrm{MeV}$ range. Here we 
a)

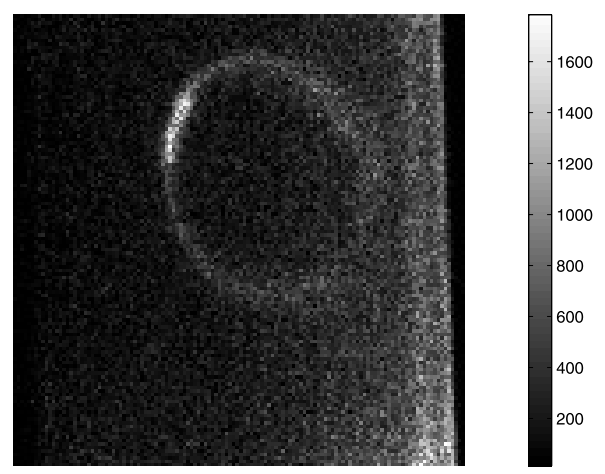

b)

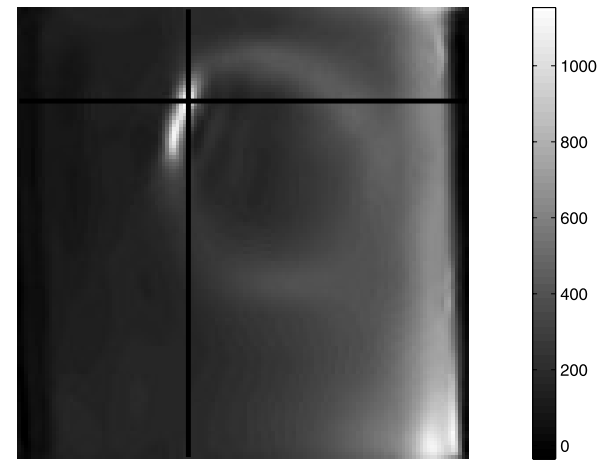

Figure 1. SI12. (a) Signal and (b) Processed Signal on December 22, 2000 (14:23:44 UT) Raw SI12 image. (b) Wavelet Denoised SI12 image. The cross hairs mark the spatial location of the onset.

used fluxes measured by SOPA on five different satellites: 1989-046, 1994-084, LANL-01A, 1991-080. We consider only four proton channels for our study: 50-75, 75-113, 113-170, and 170-250 keV.

[6] The NASA-IMAGE satellite was launched on the 25th of March, 2000 to study magnetospheric phenomena through remote-sensing using FUV and EUV radiation, radio waves and energetic neutral atoms [Burch, 2000]. One of the two FUV channels of the Spectrographic Imager, the SI12 channel, images Doppler shifted Lyman $\alpha$ radiation to monitor the global scale proton precipitation [Mende et al., 2000]. The SI12 removes the intense ( $>10 \mathrm{kR})$ geocoronal Lyman $\alpha$ background by spectral filtering which would otherwise appear as an impenetrable diffuse glow.

[7] The IMAGE FUV SI-12 instrument measures the Lyman $\alpha$ photon flux produced by energetic charge exchanged hydrogen atoms, which is strongly dependent on the charge exchange cross-section. The cross-section peaks at a relatively low proton energy and the observed Lyman $\alpha$ is predominantly produced by lower energy protons after their energy had been degraded by collisions. Thus the SI12 channel signal is more directly proportional to the proton flux and provides little information about energy. From in situ satellite (DMSP) measurements the bulk of the nightside precipitating protons are in the $5-20 \mathrm{keV}$ range [Hardy et al., 1989, and references therein]. Thus it is reasonable to use the SI-12 intensity to represent a precipitating flux of protons with mean energy in the $\sim 10 \mathrm{keV}$ range.

[8] From the IMAGE data both electron and proton auroras are observed to intensify simultaneously at the same geographic location. Intense electron auroras are generally produced by quasi-dc electric potential located above the ionosphere and may not be directly related to electron injection seen at synchronous altitude where protons and electrons injections are not necessarily collocated or simultaneous [Reeves, 1998]. Nightside protons are mostly $\sim 10 \mathrm{keV}$ and therefore less affected by these few $\mathrm{keV}$ potentials and are expected to provide a better image of magnetospheric injections.

[9] After the sudden substorm enhancement of charged particles near magnetic midnight the drift trajectory of the injected protons is determined by their charge, pitch angle, and energy. Neglecting the effects of the electric field, electrons would drift eastward, and protons westward with speeds depending on pitch angle and energy. Injected particles with small pitch angles precipitate; while particles, with larger pitch angles will mirror between the hemispheres. In this work we are primarily concerned with the azimuthal or local time displacement of the plasma. The footprints of the LANL satellites (located at $6.6 \mathrm{Re}$ ) is assumed to be in the latitude range from $66.6^{\circ}$ and $67.6^{\circ}$ magnetic latitude and the sudden onset of precipitation seen by SI12 at these latitudes should correspond to a flux enhancement visible to the LANL satellites.

\section{Comparison With Drift Model}

[10] The drift paths and drift times of protons are calculated using the test-particle code of Delcourt et al. [1990]. We modeled the earth's magnetic field with Tsyganenko 89 [Tsyganenko, 1989] using the appropriate $\mathrm{K}_{\mathrm{p}}$ and the electric field with Volland [Volland, 1978]. Eleven substorms were selected in the years 2000 and 2001 for this study based on the following criterion. There had to be at least one LANL satellite with at least one energy channel that measured a factor of ten change within a twenty minute period. In many cases multiple satellites had multiple channels that satisfied this condition. These enhancements gave easily identifiable onset times. In events where the optical intensification was not accompanied by an identifiable flux enhancement at the LANL satellites, the injection region presumably did not reach the 6.6 Re latitude region and our study is therefore restricted to investigating only those events that intersect the $6.6 \mathrm{R}_{\mathrm{e}}$ region.

[11] The SI12 measurement identified the onset time ( $\left.\mathrm{T}_{\text {aurora }}\right)$ with an accuracy permitted by the 5 second exposure and two minute cadence of the IMAGE FUV experiment and magnetic local time of an injection. The de-noising function in the Rice Wavelet Toolkit (http:// www.dsp.rice.edu/software/RWT) was used to remove noise from raw SI12 images. The magnetic local time of the resulting global maximum in the de-noised image was taken to be the injection's magnetic local time coordinate illustrated by cross hairs (Figure 1). This selection rule provides a simple criterion to process consistently and objectively a large batch of data and pick a single location that can be used in the computations. The example shown in Figure 1 was selected at random and it shows that in the example the protons showed a significant local time extent in the onset frame. The region indicated by the cross hairs was picked by the technique described above, it is interesting to note that this location was the same as a uniquely identifiable most 


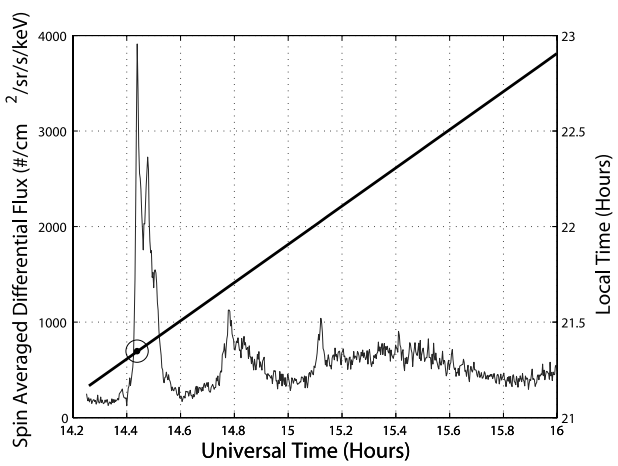

Figure 2. 1994-084: Example of the determination of the time of proton flux $(75-113 \mathrm{keV})$ enhancement December $22,2000$.

intense onset region in the electron aurora as seen by IMAGE WIC.

[12] For each LANL satellite and energy channel that satisfied the above criterion, the time of sudden enhancement and the satellite local time coordinate at the corresponding enhancement time was recorded. We used local time instead of the magnetic local time since there are only minor differences between the two. On Figure 2 we plotted the proton flux in the $(75-113 \mathrm{keV})$ channel. We have overplotted the satellite location in local time with the scale on the right. The circle on the plot indicates the LANL satellite location in local time at the time of the enhancement. The actual drift time from injection to LANL satellite is taken to be the time difference between the injection time $\left(\mathrm{T}_{\text {sat }}\right)$ and the enhancement time $\left(\mathrm{T}_{\text {aurora }}\right)$. The selected substorms provided a wide range of distances to test our observations against the models.

[13] Proton drift trajectories were generated within our assumed field models. We assigned final conditions so that protons ended up at the satellite's recorded local time coordinate at radial distance of $6.6 \mathrm{R}_{\mathrm{E}}$ with one of four different energies: $62.5,94.0,141.5,210.0 \mathrm{keV}$. These are the mean values of the energy ranges of the four selected LANL energy channels. Protons were also assigned one of six different pitch angles: $20^{\circ}, 30^{\circ}, 40^{\circ}, 50^{\circ}, 60^{\circ}, 70^{\circ}$. We simulated the proton trajectory in reverse time until the proton reached the recorded magnetic local time of the injection. These predicted travel times are plotted against the measured travel times $\left(\mathrm{T}_{\text {sat }}-\mathrm{T}_{\text {aurora }}\right)$ in the scatter plots (Figure 3). The circles are centered on the mean predicted travel time for the different pitch angles; the maximum and minimum predicted travel times for the different pitch angles are marked by the triangles.

\section{Discussion}

[14] A total of 60 data points were plotted for all four energy channels, and the correlation coefficient between the mean predicted and the actual travel times was 0.79 . This is relatively good agreement considering the uncertainties. The lowest energy channel $(50-75 \mathrm{keV})$ has considerable instrument noise due to temperature dependent "dark currents" and the derived arrival times will be subject to greater errors. The precipitation regions have widths of up to a few hours local time. We should expect a spread of arrival times around the chosen single magnetic latitude since the entire injection region introduces energetic particles and the 2D local maximum seen in SI12 data need not necessarily correspond exactly with the 1D local maximum in LANL data. Additionally, the LANL measurements track the flux of protons within a range of energies. The enhancement seen in the LANL data represents a mix of proton energies with a scatter around the modeled trajectory the mean energy of the channel.

[15] As discussed previously the optical measurement of proton precipitation through Lyman alpha detection emphasizes protons of lower energy $(>2 \mathrm{keV}$ and $<10 \mathrm{keV}$ ) protons whereas the LANL synchronous altitude particle measurements, are responsive to particles in the higher energy range. However if the high and low energy particles had been injected simultaneously at the same place as seen by IMAGE then our analysis of the drift of the higher energy particles using the Los Alamos data is valid. It should be noted that protons in the energy range of $>50 \mathrm{keV}$ are mainly driven by gradient and curvature drift and the choice of the electric field model has minimal significance. The validation of the model with observational data shows that substorm related proton enhancement is indeed coupled to the proton injection in the magnetosphere.

\section{Conclusion}

[16] The energetic protons observed by the LANL satellite show sudden flux increases in response to particle injection associated with substorms as seen by the IMAGE satellite FUV instrument. The progressive delay of the observation of the lower energy particles, i.e., dispersion, is quantitatively consistent with the model of sudden injection of all particles at onset and the subsequent azimuthal drift of protons from the injection point to the satellites in a quasi-static magnetic and electric field. The study was restricted to events where the particle injection reaches latitude regions that are conjugate to geosynchro-
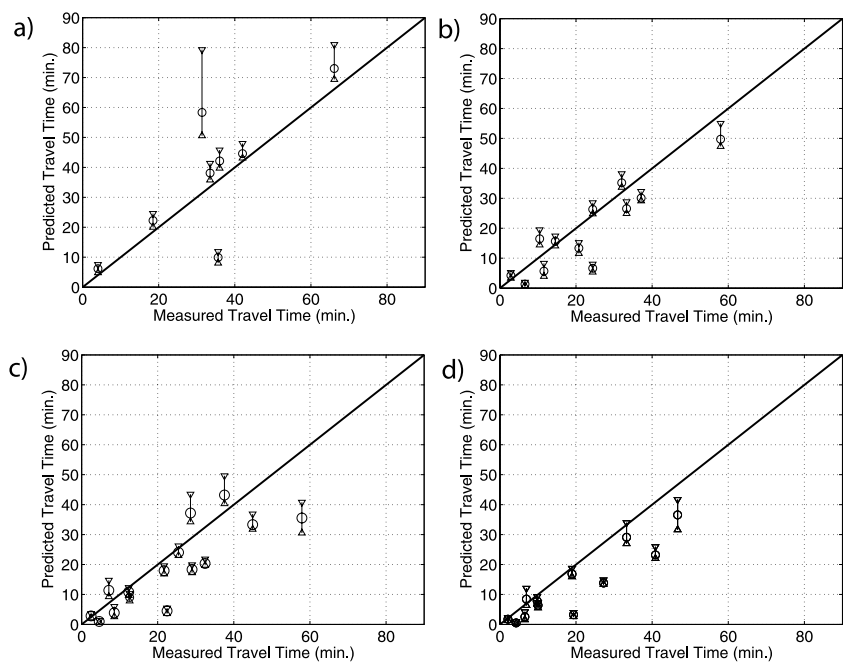

Figure 3. Scatter plots of the Predicted Travel Time $\left(\mathrm{T}_{\text {calc }}\right)$ versus Measured Travel Times $\left(\mathrm{T}_{\text {sat }}-\mathrm{T}_{\text {aurora }}\right)$ at different particle energies. (a) $62.5 \mathrm{keV}$. (b) $94.0 \mathrm{keV}$. (c) $141.5 \mathrm{keV}$. (d) $210.0 \mathrm{keV}$. 
nous satellites at $6.6 \mathrm{Re}$ radial distance. The high energy particles show greater consistency between the observed and model calculated value of their travel time from the injection point to the satellite.

[17] The observed consistency suggests that the proton auroral intensification at substorm onset and the proton injection in the magnetosphere occur simultaneously on the same MLT (longitude region). Since substorm onsets appear spatially localized the injection regions may also be localized and confined in local time. The observed consistency also validates the models used in the study and their underlying assumptions.

[18] Acknowledgment. IMAGE FUV analysis is supported by NASA through Southwest Research Institute subcontract 83820 at the University of California, Berkeley, contract NAS5-96020.

\section{References}

Birn, J., M. F. Thomsen, J. E. Borovsky, G. D. Reeves, D. J. McComas, R. D. Belian, and M. Hesse (1998), Substorm electron injections: Geosynchronous observations and test particle simulations, J. Geophys. Res., 103, 9235.

Burch, J. L. (2000), IMAGE mission overview, Space Sci. Rev, 91, 1.

DeForest, S. E., and C. E. McIlwain (1971), Plasma clouds in the magnetosphere, J. Geophys. Res., 76, 3587.

Delcourt, D. C., J.-A. Sauvaud, and A. Pedersen (1990), Dynamics of single-particle orbits during substorm expansion phase, J. Geophys. Res., 95, 20,853.

Hardy, D. A., M. S. Gussenhoven, and D. Brautigam (1989), A statistical model of ion precipitation, J. Geophys. Res., 94, 370.

Hones, E. W. (1979), Transient phenomena in the magnetotail and their relation to substorms, Space Sci. Rev., 23, 393.

Li, X., D. N. Baker, M. Temerin, G. D. Reeves, and R. D. Belian (1998), Simulation of dispersionless injections and drift echoes of energetic electrons associated with substorms Geophys, Res. Lett., 25(20), 3763 .
Lopez, R. E., D. G. Sibeck, R. W. McEntire, and S. M. Krimigis (1990), The energetic ion substorm injection boundary, J. Geophys. Res., 95, 109.

Lui, A. T. Y. (1988), What is a magnetospheric substorm expansion made of?, Eos Trans. AGU, 69, 435.

Mauk, B. H., and C. I. Meng (1983), Dynamical injections as the source of near geostationary quiet time particle spatial boundaries, $J$ Geophys. Res., $88,10,011$.

McIlwain, C. E. (1974), Substorm injection boundaries, in Magnetospheric Physics, edited by B. M. McCormac, pp. 143-154, Springer, New York. Mende, S. B., et al. (2000), Far ultraviolet imaging from the IMAGE space-

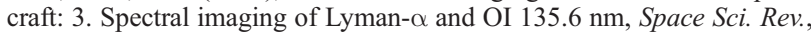
91, 287.

Mende, S. B., H. U. Frey, B. J. Morsony, and T. J. Immel (2003), Statistical behavior of proton and electron auroras during substorms, J. Geophys. Res., 108(A9), 1339, doi:10.1029/2002JA009751.

Nakamura, R., W. Baumjohann, M. Brittnacher, V. A. Sergeev, M. Kubyshkina, T. Mukai, and K. Liou (2001), Flow bursts and auroral activations: Onset timing and foot point location, J Geophys. Res., 106, 10,777.

Reeves, G. D. (1998), New perspectives on substorm injections, paper presented at Fourth International Conference on Substorms, Sol.-Terr. Environ. Lab., Hamanako, Japan.

Reeves, G. D., R. D. Belian, and R. A. Fritz (1991), Numerical tracing of energetic particle drifts in a model magnetosphere, J. Geophys. Res., 96, $13,997$.

Reeves, G. D., G. Kettmann, T. A. Fritz, and R. D. Belian (1992), Further investigation of the CDAW 7 substorm using geosynchronous particle data: Multiple injections and their implications, J. Geophys. Res., 97, 6417.

Tsyganenko, N. A. (1989), A magnetospheric magnetic field model with a warped tail current sheet, Planet. Space Sci., 37, 5.

Volland, H. (1978), A model of the magnetospheric electric convection field, J. Geophys. Res., 83(12), 2695.

E. C. Chi and S. B. Mende, Space Sciences Laboratory, University of California, Berkeley, Centennial Drive at Grizzly Peak Boulevard, Berkeley, CA 94720-7450, USA. (mende@ssl.berkeley.edu)

M.-C. Fok, NASA Goddard Space Flight Center, Greenbelt, MD 20771, USA.

G. D. Reeves, Los Alamos National Laboratory, Los Alamos, NM 87545, 